\title{
PREOCUPAÇÕES INERENTES NA APLICAÇÃO DAS METODOLOGIAS ATIVAS NAS GRADUAÇÕES EM ENGENHARIA
}

Rogério Bastos Quirino - rb_quirino@hotmail.com

Universidade do Estado de Mato Grosso, Faculdade de Ciências Exatas e Tecnológicas Avenida dos Ingás, 3001

CEP 78555000 - Sinop - Mato Grosso

Gilmar Barreto-gbarreto@dsif.fee.unicamp.br

Faculdade de Engenharia Elétrica e de Computação, Universidade Estadual de Campinas Av. Albert Einstein, 400, Cidade Universitária Zeferino Vaz

CEP - 13083-285 - Campinas - SP

José Tarcísio Franco de Camargo - jtfc@bol.com.br

Centro Regional Universitário de Espírito Santo do Pinhal, UNIPINHAL

Av. Hélio Vergueiro Leite, $s / n$ - Jardim Universitário

CEP - 13990-000 - Espírito Santo do Pinhal - SP

Resumo: A demanda por profissionais das áreas de engenharia vem adquirindo novos contornos nos últimos anos e especialmente com as implantações das novas DCNs e os recentes avanços nas tecnologias digitais, com reflexos diretos na formação universitária desses futuros profissionais. Envolvidas nesse contexto, estão as tendências como a hiper conectividade, as atividades virtuais e a maior inserção de tecnologia e digitalização no cotidiano, que se intensificaram com força na pandemia vigente da covid-19, incorporando juntamente a implantação das metodologias ativas consensual e imperativa, vêm impulsionando avanços, novos desafios, inéditas possibilidades de aperfeiçoamento de ensino-aprendizagem, trabalho e estudo, mas também ainda demandando muitas reflexões. Baseado neste cenário vigente, este trabalho procura discutir sobre os problemas intrínsecos da estruturação de aulas a partir de modelos de currículos flexíveis com integração das disciplinas, projetos integradores, $e$ principalmente, das metodologias ativas que tiram o foco do professor e dão mais autonomia aos alunos, ressaltando predominantemente os aspectos raramente explicitados na literatura do que deveria de fato constituir o núcleo da metodologia ativa, ou seja, o real exercício da docência em filosofia no sentido estrito da palavra, escopo e olhar por vários prismas do conteúdo das disciplinas e relações interdisciplinares na construção, maturação e real absorção e utilização do conhecimento, independentemente da metodologia ativa utilizada. Cabe ressaltar que pouco ou nenhum empenho e reconhecimento da necessidade dessa discussão pontual importantíssima, meramente deslocará o problema já existente na metodologia tradicional às metodologias em voga.

Palavras-chave: Metodologia Ativa. Sistemas 4.0. Taxonomia de Bloom. Ensino de Engenharia. 


\section{INTRODUÇÃO}

As mudanças que vêm ocorrendo nas demandas sociais rompem fronteiras entre áreas do conhecimento e passam a exigir, além de sólida formação teórico-prática nas modalidades, autonomia e criatividade por parte dos profissionais e dos cursos de engenharia. Neste contexto, é pouco visitado o aspecto da docência com a profundidade necessária sobre "o que" e "como" estudar no ensino de engenharia, mesmo nas modernas metodologias ativas. Muito além de dominar os conteúdos da engenharia, o mercado de trabalho exige um altíssimo grau de autonomia, criatividade e visão holística, para que o profissional egresso de um curso de engenharia possa transitar entre as áreas do conhecimento, trabalhar colaborativamente com empenho e dar sentido às demandas sistêmicas, de acordo com as necessidades sociais.

De forma recorrente, a formação do engenheiro no Brasil tem contemplado principalmente a transmissão fria, crua e não processada de fundamentos básicos, que pouco evoluem e mesmo assim nem sempre são estudados de forma adequada. Este cenário, por sua vez, choca-se com as tecnologias do momento e com as soluções esperadas para problemas existentes na engenharia, comprometendo a atuação do engenheiro recém-formado quando exposto ao novo e à necessidade de autonomia para inovação e solução de problemas.

Como agravante, as evoluções cada vez mais rápidas no setor tecnológico e o consequente aumento na complexidade dos problemas, tem forçado o ensino em engenharia a se tornar cada vez menos estruturado e transparente. Pode-se observar, neste sentido, que os cursos das engenharias têm adotado o uso de verdadeiras "caixas-pretas" em suas aulas, com o intuito de compensar a velocidade em que ocorrem as mudanças.

É neste contexto que este trabalho pretende provocar reflexões quanto à necessidade de mudanças no paradigma atualmente dominante no ensino de engenharia. As reflexões propostas pretendem mostrar a necessidade de adequações nos cursos de engenharia, de forma a minimizar o risco de o profissional ficar rapidamente defasado poucos anos após sua formação.

Da mesma forma, também é necessário refletir a respeito da estrutura das instituições de ensino em engenharia, tal que elas se tornem capazes de se reinventar, vindo a vislumbrar uma realidade pouco discutida na formação do profissional, tornando-se plenamente aptas a promover uma formação adequada a este novo cenário que se apresenta.

\section{HISTÓRICO DE ESTUDOS}

Na prática, tal problematização é considerada na elaboração dos modelos de currículos flexíveis com integração das disciplinas, projetos integradores e metodologias ativas, que tiram o foco do professor e dão mais autonomia aos alunos, e desempenham papel fundamental na formação da base piramidal na taxonomia de Bloom (Figura1). É exatamente na aplicação das metodologias ativas, que se encontra a gênese do processo de formação profissional, e que nela pode ser camuflada uma realidade que quase nunca vem à tona. Como implementar as metodologias ativas de forma eficiente se a falha central, em teoria, já existente na denominada metodologia tradicional de berço (aula expositiva) é simplesmente transferida de lugar? Tal falha sucede o método tradicional, em que já estava contida, razão pela qual o método tradicional (aula expositiva) venha a ser mal avaliado, subjugado por gerar sentimento de aprendizagem equivocado:

"Os alunos em salas de aulas ativas aprenderam mais (como seria de esperar com base em investigação prévia), mas a sua percepção da aprendizagem, enquanto 
positivo, era inferior ao dos seus pares em ambientes passivos. Isto sugere que as tentativas de avaliar a instrução com base sobre a percepção que os alunos têm da aprendizagem, pode inadvertidamente, promover métodos pedagógicos (passivos) inferiores. Por exemplo, um docente superestrela poderia criar um sentimento de aprendizagem tão positivo que os alunos escolheriam essas aulas em vez da aprendizagem ativa", (DESLAURIERSA, L., 2019).

Exatamente daí decorre o fato de que na aula expositiva do docente superestrela certamente não é feito o exercício de filosofia no sentido estrito da palavra, escopo e abstração para abarcar com profundidade no que e como estudar na disciplinaridade e interdisciplinaridade, erro que poderá ser igualmente repetido na aplicação dos métodos ativos. Significa dizer que os métodos ativos, por si só, não se constituem meios que naturalmente já provêm a correção dessa falha. A falha não é consequência somente da percepção pobre que os alunos têm da aprendizagem, mas também da ação pobre do docente, independentemente da metodologia empregada. Essa falha poderia ser naturalmente importada, o que conduziria a uma falsa impressão de que os degraus da pirâmide da taxonomia de Bloom (Figura 1) estejam sendo consistentemente construídos.

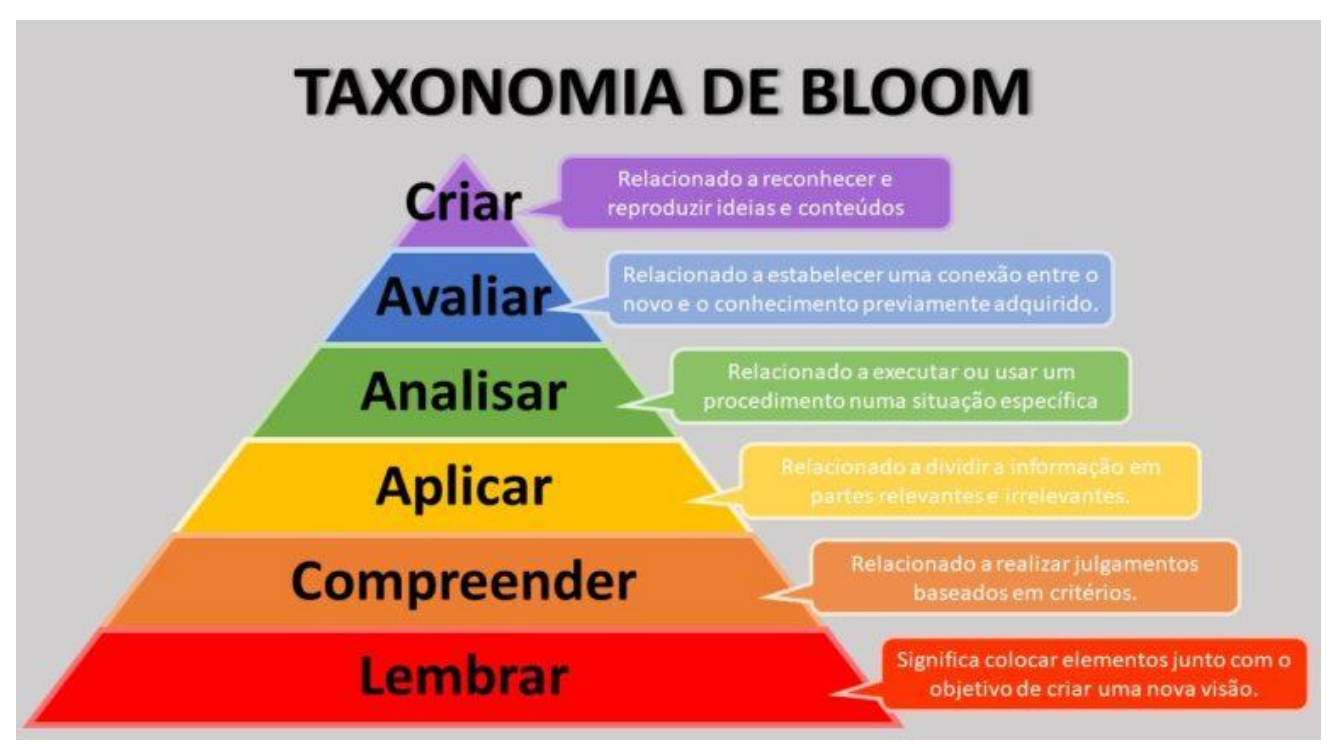

Fonte: Augusto, M., 2020.

O exercício docente ativo cumpre um papel importante de idealização e balizamento referenciais, de caminho de realimentação e avaliação do protagonismo na relação aluno/docente, quaisquer que sejam as metodologias empregadas. Tal falta de ação docente tem reflexos negativos e não pode ser simplesmente carregada sem que a demos um tratamento adequado, sem o qual, mesmo com o emprego das metodologias ativas, os resultados serão ineficazes do ponto de vista da essência da formação e domínio do conhecimento.

Ainda no bojo desse processo, existe um agravante que reside no fato de que há muito tempo o sistema de ensino de graduação de engenharia brasileiro, com o intuito de se adequar a era 4.0, que requer do profissional o cumprimento da fase topo da pirâmide de Bloom (Figura 
1), vem implementando abordagens de ensino baseadas na filosofia "caixas-pretas", inerentes e constituintes das plataformas dos sistemas 4.0, também tópico da atual de discussão no ensino de engenharia, tratado em (DE OLIVEIRA,2020).

Nessas abordagens, a filosofia de ensino é baseada quase somente na utilização premente de pacotes Matlab, Scilab, Octave, Mathcad, Simulink, entre outros..., passíveis de mera alimentação de dados, e de posterior devolução de resultados para análise, sem que o acadêmico tenha a compreensão teórica em profundidade suficiente do cerne sobre como o pacote pode ser implementado. Um exemplo, pode ser dado pela não tão simples multiplicação das matrizes $\mathrm{A}$ e $\mathrm{B}$ para obter a matriz $\mathrm{C}$ podemos fazer esta operação de seis maneiras distintas, (Barreto, 2002). A estrutura usada pelo Matlab para fazer esta operação não está disponível e é aceita passivamente por todos os usuários do software.

Deste modo a teoria ou método matemático em que o software está baseado ficando para o aluno apenas como programá-lo, prejudica significativamente a formação desses acadêmicos ao desenvolvimento, manutenção e utilização adequada de tais pacotes, indispensáveis à formação competente para inserção profissional na era 4.0, tornando-os meros "pilotos de programas" com conhecimentos teóricos superficiais, sem abstrações e reflexões maduras em profundidade sobre o conteúdo, despreparados ao desenvolvimento, e modificação de tais sistemas.

Durante décadas, há evidências de que aprendizagem ativa - técnicas de sala de aula projetado para levar os alunos a participar do processo de aprendizagem - produz melhores resultados educacionais para estudantes em praticamente todos os níveis pode ser questionada pelos argumentos anteriores.

Figura 2- Candidatos X Concluintes em Engenharia em 2017.

O funil da Formação em Engenharia (Estimativa)

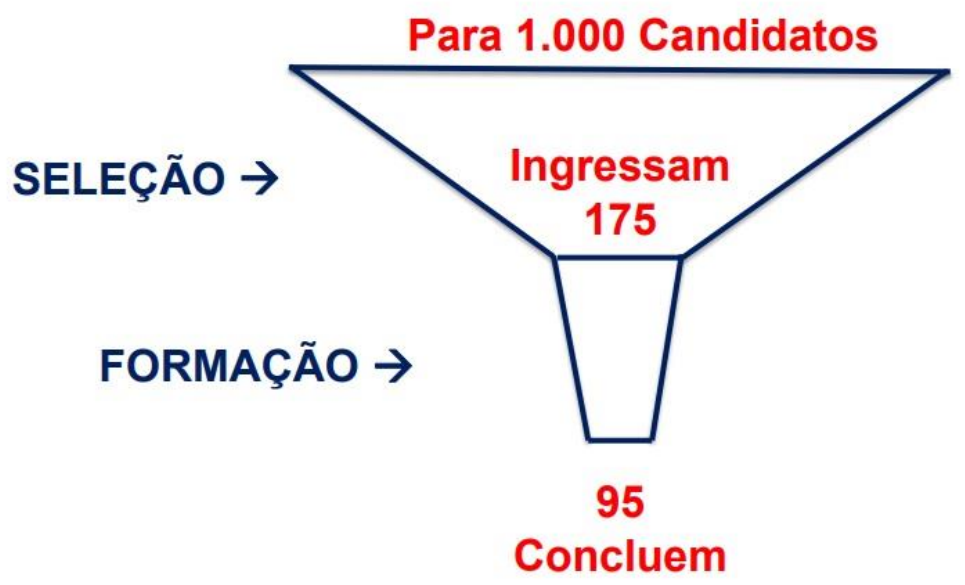

Fonte: Organizado por Vanderli Fava de Oliveira. Base: dados inep.gov.br, set/2017

O Prof. Vanderli ressalta em sua apresentação o número de Candidatos X Concluintes em Engenharia em 2017, Figura 2, (OLIVEIRA, 2020.) Infelizmente destes 95 concluintes muitos irão atuar em atividades não relacionadas a engenharia e uma das causas deste funil pode ter como causa a falta de ir nas bases fundamentais da teoria o que dificulta o aprendizado. 
Entretanto movimentos tem surgido já no ensino médio para atacar este problema, aqui podemos destacar as olimpíadas de matemática, astronomia e computação entre outras tantas em que o material utilizado muitas vezes é papel e lápis, o que nos dá esperança que este quadro pode ser revertido.

Deste modo esperamos que a implantação das novas DCNs - Diretrizes Curriculares Nacionais em Engenharia, que incentiva a competência através de práticas laboratoriais e reais no lugar de se ministrar somente conteúdo possa reverter este quadro.

\section{METODOLOGIA}

A metodologia empregada procura levantar e evidenciar parte dos problemas inerentes nas relações de didáticas, conteúdos e aprofundamentos utilizados no ensino que podem se prostrar como geradores de inconsistências ou oportunidades singulares na formação profissional à aquisição de competências ao entendimento, operação e criação de sistemas da era 4.0, tendo como cerne da discussão a implementação das metodologias ativas tratada na nova lei de diretrizes e bases (OLIVEIRA,2020). A didática, o conteúdo propriamente dito, a profundidade e a forma de seu tratamento, foram desenvolvidos de forma a maximizar a capacitação científico-tecnológica e de inovações, da produtividade e competitividade dos alunos no mercado em nível global, mas antes de tudo, nos efeitos da resposta efetiva, coerente, da implementação das metodologias ativas, gênese da formação profissional. Ademais, a metodologia procurou criar alguns caminhos a serem trilhados com base em alguns trabalhos de fundo didáticos desenvolvidos em disciplinas de graduação em engenharia elétrica em instituições pública e privada.

Mais especificamente, encontra-se em fase de desenvolvimento uma pesquisa qualitativa no curso de bacharelado em engenharia elétrica da Unemat, para o aprofundamento da questão e para melhor subsidiar as proposições dela decorrentes, utilizando vasto referencial bibliográfico clássico e contemporâneo, bem como uma vasta gama de experiências de ensino em duas disciplinas curriculares, que fomentam a discussão, analisando aspectos que demonstram riscos ou oportunidades reais à formação profissional sob o cenário atual em que as metodologias ativas são amplamente discutidas. Mais especificamente, as disciplinas de Sinais e Sistemas e Sistemas de Controle Automático, do $4^{\circ}$ e $6^{\circ}$ semestres, respectivamente, do curso de engenharia elétrica da Unemat, Campus de Sinop, estão sendo utilizadas como plataformas ao desenvolvimento da pesquisa. A pesquisa avalia qualitativamente os níveis de dificuldade, causas e efeitos ao entendimento e desenvolvimento integral de um projeto melhorado ou análogo ao de um laboratório virtual ao ensino de modelagem e dinâmica de sistemas lineares desenvolvido em (RODRIGUES,2020; SILVA,2012), à criação de um pacote computacional de apoio didático aos estudos realizados em (ANDRADE,2016; ROSSET,2018), bem como a exploração de códigos fonte de pacotes computacionais ("toolboxes") à investigação de métodos matemático-numéricos, tanto para os ambientes computacionais Unix como Windows.

\section{DISCUSSÕES E RESULTADOS}

Dada interrupção do período letivo acadêmico em decorrência da pandemia, já é possível observar uma tendência de os alunos com conhecimentos teóricos mais aprofundados, em escopo e filosofia, nas disciplinas do curso, e com maior domínio das relações 
interdisciplinares, principalmente envolvendo a tecnologia da informação, apresentaram um melhor desempenho ao entendimento, melhoramento e desenvolvimento de pacotes relacionados as referências (RODRIGUES,2020; SILVA,2012; ANDRADE,2016; ROSSET,2018). A fim de estender a discussão, tomemos como exemplo a utilização de "toolboxes" de controle das plataformas de computação científica Matlab ou Scilab, ao ensino da disciplina de teoria de controle clássico e moderno nos cursos de engenharia elétrica. Em geral, os docentes que ministram tal disciplina lançam mão de "toolboxes" nesses ambientes, sem que o aluno tenha visto em profundidade, entendido e resolvido de próprio punho, 'a priori', de forma minimamente satisfatória, por exemplo, a solução de equações diferenciais lineares como respostas a determinadas entradas ou equações diferenciais lineares homogêneas para um dada condição inicial, através de ferramentas matemáticas como transformada de Laplace ou a teoria de autovalores e autovetores da álgebra matricial. Como extensão, os exemplos poderiam compreender teorias relacionadas às disciplinas de redes neurais, processamento digital de sinais, identificação de sistemas, controle robusto, etc, (QUIRINO, BARRETO e CLAUDY, 2019).

Um trabalho de interesse sobre este tema abordando contextos motivacionais indica que os alunos de engenharia do primeiro ano de um ambiente metodologias ativas, e com base em suas próprias descrições do que eles consideram ser um bom dia de estudo, são motivados por processos de colaboração e relações sociais, e ainda mais a sensação de dominar algo novo, mais do que por perspectivas futuras ou o próprio conhecimento do assunto. Isso é importante para nós como supervisores e professores no primeiro ano para saber e levar em consideração quando pretendemos motivar o nosso alunos, ( BØGELUND e NØRGAARD, 2019).

As conclusões desta pesquisa, certamente revelarão aspectos importantes plausíveis que os formandos de engenharia certamente terão de lidar e trabalhar durante sua formação profissional, se desejarem de fato se colocarem como desenvolvedores de tecnologias dos sistemas modernos. Caso contrário, serão meramente usuários e aplicadores de tecnologias "caixas-pretas", que poucos dominam, modificam e desenvolvem.

\section{CONSIDERAÇÕES FINAIS}

Com o intuito de melhor validar as conclusões e criar um modelo de estudo de caso de referência ("benchmark") que permita estudar e comparar nuances da formação dos estudantes de engenharia no contexto de ensino aqui discutido, estamos procurando firmar um acordo ao uso das instalações e recursos didáticos de treinamento do SENAI/Sinop-MT, por não dispormos ainda de laboratórios satisfatoriamente bem equipados, a fim de se criar um "benchmark" ao projeto de modificação, controle e operação de planta ou dispositivo, empregando de forma mais abrangente possível as tecnologias de última geração, subsidiado com os conhecimentos teórico-práticos adquiridos nas disciplinas dos cursos de bacharelado em engenharia elétrica da UNEMAT/Sinop.

Propomos aos docentes de instituições de ensino que dispõem de laboratórios com instalações de equipamentos com tecnologia de ponta, e que se coadunam com a provocação e idéia aqui lançadas, que investiguem estas nuances de formação, sob o referencial da proposta aqui elaborada, de forma a procurar dar uma resposta ao aqui delineado.

Cabe ressaltar que neste trabalho estamos procurando demonstrar a existência de uma falha no percurso de evolução da didática de ensino, que certamente se corroborada, será certamente tratada e corrigida, em prol, há muito tempo, do carente e deficiente sistema de ensino de graduação em engenharia. 


\section{REFERÊNCIAS}

ANDRADE, T. G. e QUIRINO, R. B. . Um Estudo Unificado das Teorias de Estabilidade de Sistemas Lineares de Controle Automático. Congresso Técnico Científico da Engenharia e da Agronomia - CONTECC'2016,-- Foz do Iguaçu, PR, 2016.

AUGUSTO, M.. Será viável falar-se em "ensino" de Empreendedorismo? . Disponível em: $<$ https://engenhariae.com.br/editorial/engenharia-em-pauta/sera-viavel-falar-se-em-ensino-deempreendedorismo-segunda-parte> Acesso em 20/06/2020.

BARRETO, G.. Modelagem Computacional Distribuída e Paralela de Sistemas e de Séries Temporais Multivariáveis no Espaço de Estado. 2002. 401p. Tese (doutorado) - Universidade Estadual de Campinas, Faculdade de Engenharia Elétrica e de Computação, Campinas, SP. Disponível em: <http://www.repositorio.unicamp.br/handle/REPOSIP/261255>. Acesso em 1 ago. 2018.

BØGELUND, P. e NØRGAARD, B.. First-year engineering students' perception of what makes a good study day . Proceedings of the SEFI 47th Annual Conference, p.125-134, 2019.

DESLAURIERSA, L.; MCCARTYA L. S. ; MILLERC K. ; CALLAGHANA K. , AND KESTINA G.. Measuring Actual Learning versus Feeling of Learning in Response to being Actively Engaged in the Classroom PNAS | September 24, 2019 | vol. 116 | no. 39 | 1925119257 <https://www.pnas.org/content/116/39/19251> Acesso em 12/04/2020.

OLIVEIRA, V.F.. A Engenharia e as Novas DCNs - Oportunidades para Formar Mais e Melhores Engenheiros, $1^{\text {a }}$ Edição, LTC, 2019.

OLIVEIRA, V.F. A Engenharia e as Novas DCNs - Oportunidades para Formar Mais e Melhores Engenheiros. Palestra. Disponível em: $<$ https://www.youtube.com/watch?v=OEQlvky2u4U > Acesso em 22/06/2020.

QUIRINO, R. B. ; BARRETO, G. ; ClAUDY, V.R.S. . A Era dos Sistemas 4.O: Contribuição ou Comprometimento ao Ensino de Graduação No Brasil? VII Seminário Inovações Curriculares, Campinas, SP., 2019.

RODRIGUES, D.A.; QUIRINO R.B.; GASOTTO, M.A. Development of a Public Domain Virtual Laboratory for the Interdisciplinary Study of Dynamic Systems. Brazilian Journals of Development, Curitiba, v. 6, n. 7, p.52390-52393, ISSN 25258761, julho, 2020.

ROSSET, K. R.; QUIRINO, R. B.; SOBRINHO, L. M. M. Anti-Transformada de Laplace Versus Integral de Convolução na Obtenção de Respostas de Sistemas Lineares - Um Estudo de Caso Didático. XLIV Congresso Brasileiro de Educação em Engenharia COBENGE 2018, Salvador, BA, 2018.

SILVA, E. A. ; QUIRINO, R. B. ; GASOTTO, M. A. Ambiente Computacional Didático ao Estudo de Sistemas Dinâmicos em Engenharia. Seminário de Iniciação Cientifica e 
Tecnologia da UTFPR, Curitiba - PR. Programa executável denominado controllab, de domínio público, depositado no sítio www.sourceforge.org, 2012.

\title{
INHERENT CONCERNS IN THE APPLICATION OF ACTIVE METHODOLOGIES IN ENGINEERING GRADUATIONS
}

\begin{abstract}
The demand for professionals in the engineering areas has been acquiring new contours in recent years and especially with the implantations of the new DCNs and the recent advances in digital technologies, with direct effects on the university education of these future professionals. Involved in this context are trends such as hyper connectivity, virtual activities and the greater insertion of technology and digitalization in daily life, which have intensified intensely in the current pandemic of covid-19, together incorporating the implementation of consensual and imperative active methodologies, they have been driving advances, new challenges, unprecedented possibilities for improving teaching-learning, work and study, but also still demanding many reflections. Based on this current scenario, this paper seeks to discuss the intrinsic problems of structuring classes based on flexible curriculum models with integration of disciplines, integrative projects, and mainly, of active methodologies that take the focus of the teacher and give more autonomy to students, predominantly emphasizing the aspects rarely explained in the literature of what should in fact constitute the nucleus of the active methodology, that is, the real exercise of teaching in philosophy in the strict sense of the word, scope and looking through various prisms of the content of disciplines and interdisciplinary relations in the construction, maturation and real absorption and use of knowledge, regardless of the active methodology used. It is worth mentioning that little or no commitment and recognition of the need for this very important timely discussion will merely shift the problem that already exists in the traditional methodology to the methodologies in vogue.
\end{abstract}

Keywords: Active methodology. 4.0 systems. Bloom taxonomy. Engineering education. 